\title{
Serum Golgi protein 73 is a prognostic rather than diagnostic marker in hepatocellular carcinoma
}

\author{
MIN DONG $^{1 *}$, ZHAN-HONG CHEN $^{1,2^{*}}$, XING LI $^{1 *}$, XIAO-YUN LI ${ }^{1,3}$, JING-YUN WEN $^{1}$, \\ QU LIN ${ }^{1}$, XIAO-KUN MA ${ }^{1}$, LI WEI $^{1}$, JIE CHEN $^{1}$, DAN-YUN RUAN ${ }^{1}$, ZE-XIAO LIN $^{1}$, \\ TIAN-TIAN WANG ${ }^{1}$, DONG-HAO WU ${ }^{1}$ and XIANG-YUAN WU ${ }^{1}$
}

${ }^{1}$ Department of Medical Oncology and Guangdong Key Laboratory of Liver Disease, The Third Affiliated Hospital, Sun Yat-sen University, Guangzhou, Guangdong 510630; ${ }^{2}$ Department of Medical Oncology of

Sun Yat-sen University Cancer Center, State Key Laboratory of Oncology in South China, Collaborative Innovation Center for Cancer Medicine, Guangzhou, Guangdong 510060; ${ }^{3}$ Department of Medical Oncology, The First Affiliated Hospital, Henan University, Kaifeng, Henan 475001, P.R. China

Received February 4, 2016; Accepted July 3, 2017

DOI: $10.3892 / 01.2017 .6938$

\begin{abstract}
Serum Golgi protein 73 (sGP73) is a candidate diagnostic biomarker for hepatocellular carcinoma (HCC). However, current evidence of its diagnostic value is conflicting, primarily due to the small sample sizes of previous studies, and its prognostic role in $\mathrm{HCC}$ also remains unclear. In the present study, sGP73 levels in 462 patients with HCC, 186 patients with liver cirrhosis, and 83 healthy controls were evaluated using ELISA, and it was identified that the median sGP73 levels were significantly higher in the $\mathrm{HCC}(18.7 \mathrm{ng} / \mathrm{ml})$ and liver cirrhosis $(18.5 \mathrm{ng} / \mathrm{ml})$ patients than in the healthy controls $(0 \mathrm{ng} / \mathrm{ml}$; both $\mathrm{P}<0.001$ ); however, the levels did not significantly differ between the HCC and liver cirrhosis groups ( $\mathrm{P}=0.632$ ). sGP73 had an inferior sensitivity and specificity for HCC diagnosis (27.79 and $77.96 \%$, respectively) compared with $\alpha$-fetoprotein (57.36 and $90.96 \%$, respectively; $\mathrm{P}<0.001)$. In the HCC group, a high level of sGP73 was associated with aggressive
\end{abstract}

Correspondence to: Dr Xiang-Yuan Wu, Department of Medical Oncology and Guangdong Key Laboratory of Liver Disease, The Third Affiliated Hospital, Sun Yat-sen University, 600 Tianhe Road, Guangzhou, Guangdong 510630, P.R. China

E-mail:wuxiangy@mail.sysu.edu.cn

*Contributed equally

Abbreviations: AFP, $\alpha$-fetoprotein; AUC, areas under curve; BCLC, Barcelona-Clinic Liver Cancer; DFS, disease-free survival; ECOG, Eastern Cooperative Oncology Group; ELISA, enzyme-linked immunosorbent assay; GP73, Golgi protein 73; HBV, hepatitis B virus; $\mathrm{HCC}$, hepatocellular carcinoma; $\mathrm{HCV}$, hepatitis $\mathrm{C}$ virus; OS, overall survival; ROC, receiver operating characteristic; sGP73, serum Golgi protein 73

Key words: hepatocellular carcinoma, serum, Golgi protein 73, prognosis clinicopathological features and independently predicted poor overall survival $(\mathrm{OS})$ time $(\mathrm{P}<0.001)$. Additionally, in patients with resectable HCC, a high level of sGP73 was associated with significantly decreased disease-free survival $(\mathrm{P}<0.001)$ and $\mathrm{OS}$ $(\mathrm{P}=0.039)$ times compared with a low level of sGP73. This study demonstrated that sGP73 is unsuitable as a diagnostic marker for the early detection of HCC; however, it is an independent negative prognostic marker, providing a novel risk stratification factor and a potential therapeutic molecular target for HCC.

\section{Introduction}

Hepatocellular carcinoma (HCC) is one of the most common types of malignancy and the third leading cause of cancer-associated mortality globally (1). In China, the incidence of $\mathrm{HCC}$ is among the highest worldwide, with Chinese patients accounting for $55 \%$ of new HCC cases. Chronic infection with hepatitis B virus (HBV) and hepatitis C virus (HCV) is the major etiology of HCC, which often arises from a background of viral hepatitis and cirrhosis (2). Lacking specific symptoms at early stages, the majority of patients with HCC are diagnosed at advanced stages, with a low 5-year survival rate of $10.1 \%$ (3) and a high 5-year recurrence rate of $70 \%$, even following liver resection (4). Therefore, early diagnosis, early treatment and early recurrence detection are extremely important in improving the prognosis of HCC.

The most common methods used in the early detection of HCC are analysis of $\alpha$-fetoprotein (AFP) level and hepatic ultrasonography. As the most widely used serum biomarker, AFP has a high specificity of 80-94\%; however, its sensitivity is only 41-65\% (5), making it an unsatisfactory biomarker for the early detection of HCC. To improve the efficiency of early diagnosis of HCC, novel candidate serum biomarkers have emerged, including Lens culinaris agglutinin reactive AFP (6), Des- $\gamma$-carboxy prothrombin (7), $\alpha$-l-fucosidase (8), glypican-3 (9), squamous cell carcinoma antigen (10), and Golgi protein 73 (GP73) (11). Of these potential HCC serum markers, GP73 is the most promising. 
GP73, a resident type II Golgi membrane protein with a molecular weight of $73 \mathrm{kDa}$, is predominantly expressed by biliary epithelial cells in the normal liver. Aberrant expression of GP73 in hepatocytes is present in chronic liver disease, including viral and non-viral hepatitis, liver cirrhosis and HCC (12-14). Additionally, gradually increasing GP73 expression levels in tissue have been observed throughout the progression from normal liver and chronic hepatitis to HCC (15). Importantly, the C-terminal ectodomain of GP73 can be released into the circulation (16). Since Block et al (17) first identified elevated serum GP73 (sGP73) levels in patients with HCC in 2005, sGP73 has been considered to be a potential biomarker for HCC.

A number of studies have evaluated the early diagnostic value of sGP73 for HCC. Several studies in which sGP73 was detected using immunoblotting demonstrated that sGP73 levels were significantly increased in HCC compared with cirrhotic controls, with a superior sensitivity and specificity to AFP for HCC diagnosis $(11,18)$. However, as a semiquantitative and laborious test, immunoblotting is unsuitable for routine clinical practice. By contrast, enzyme-linked immunosorbent assay (ELISA) is quantitative and convenient. Although a number of studies have demonstrated higher levels of sGP73 measured by ELISA in HCC than in liver cirrhosis (19-21), no significant difference in sGP73 concentrations between HCC and cirrhosis groups was identified by other studies (22-24), and in a number of studies, the sGP73 level was even lower in HCC than in liver cirrhosis $(15,25)$. Considering the small sample size of these studies, further evaluation of ELISA-measured sGP73 in large-scale studies is required to clarify its diagnostic value in HCC. In addition, although the overexpression of GP73 in HCC tissue has been reported to be associated with aggressive behavior and poor overall survival (OS) time (14), the clinicopathological effects and prognostic role of sGP73 in HCC have not yet been characterized.

In the present study, sGP73 levels in 462 patients with $\mathrm{HCC}$, 186 patients with liver cirrhosis and 83 healthy controls were measured with ELISA, and the prognostic value of sGP73 in HCC patients was assessed. It was identified that the diagnostic value of sGP73 was inferior to AFP for HCC. However, high levels of sGP73 were associated with aggressive clinicopathological characteristics and poor OS and disease-free survival (DFS) times. Cox multivariate analysis demonstrated that sGP73 was an independent prognostic factor for OS and DFS, suggesting that sGP73 has important prognostic value in HCC.

\section{Materials and methods}

Subjects. A total of 731 subjects were enrolled at the Third Affiliated Hospital of Sun Yat-sen University (Guangzhou, China) between January 2011 and August 2013, including 462 patients with HCC (420 males and 42 females, aged 11-84 years), 186 patients with liver cirrhosis (156 males and 30 females, aged 17-80 years), and 83 healthy controls (59 males and 24 females, aged 22-73 years). The diagnosis of HCC was made either by histopathology $(n=72)$ or by two imaging modalities (ultrasound, computed tomography or magnetic resonance imaging) showing an arterial enhancing lesion, with HBV and/or HCV infection $(n=390)$. The diagnosis of liver cirrhosis was based on liver histology or clinical, laboratory and imaging evidence of hepatic decompensation or portal hypertension. The clinicopathological characteristics of the HCC patients are summarized in Table I. In the liver cirrhosis group, all patients had hepatitis B cirrhosis. This study was approved by the Ethics Committee of the Third Affiliated Hospital of Sun Yat-sen University. Informed consent was obtained from each subject.

Sample collection. A 2-ml blood sample was drawn from each subject, centrifuged at 4,000 x g for $5 \mathrm{~min}$ and then aliquoted. The serum samples were stored at $-80^{\circ} \mathrm{C}$ until testing. All the blood samples from the patients with $\mathrm{HCC}$ were collected prior to the initiation of HCC treatment.

Detection of GP73 and AFP. sGP73 was detected using ELSIA kit for Golgi protein 73 (cat. no. SEB668Hu; Cloud-Clone Corp., Houston, TX, USA). Each serum sample was diluted 20 -fold in phosphate-buffered saline, and tested according to the manufacturer's instructions. Briefly, $100 \mu \mathrm{l}$ of diluted serum sample was added to the microtiter plate well pre-coated with an antibody specific to GP73 and incubated for $2 \mathrm{~h}$ at $37^{\circ} \mathrm{C}$. Anti-IgG conjugated with biotin $(100 \mu \mathrm{l})$ was added to each well and incubated for $1 \mathrm{~h}$ at $37^{\circ} \mathrm{C}$. Then the plate was washed three times with washing buffer. Avidin conjugated to horseradish peroxidase $(100 \mu \mathrm{l})$ was added to each well and incubated for $30 \mathrm{~min}$ at $37^{\circ} \mathrm{C}$. Subsequent to washing five times, $90 \mu \mathrm{l}$ of tetramethylbenzidine substrate solution was added to each well and incubated in the dark for $15 \mathrm{~min}$. The enzyme-substrate reaction was then terminated by the addition of $50 \mu \mathrm{l}$ of sulfuric acid solution. Absorbance was measured at $450 \mathrm{~nm}$ in a microplate reader (BioTek Instruments, Inc., Winooski, VT, USA). The concentration of sGP73 in the samples was determined by comparing the optical density of the samples to the standard curve.

Serum AFP was tested using a chemiluminescent immunoassay kit (Roche Diagnostics GmbH, Mannheim, Germany) at the Clinical Diagnostic Laboratories of the Third Affiliated Hospital of Sun Yat-Sen University. The upper limit of normal AFP was $8 \mathrm{ng} / \mathrm{ml}$.

Clinical outcome assessment. Laboratory and imaging data were collected every month in patients with unresectable HCC. For patients who underwent curative treatment, radical resection or radiofrequency ablation (RFA), laboratory and imaging examination were conducted every three months after surgery or RFA. OS time was defined as the interval between the date of blood sample collection and the date of mortality from HCC, or the date of last follow-up if patients were still alive. DFS time was calculated as the interval between the date of surgery or RFA and the date of local recurrence/distant metastasis, date of mortality, or the latest date, when censored.

Statistical analysis. Quantitative values were presented as median interquartile range (IQR; 25 th and 75 th percentiles) due to the abnormal distribution of sGP73. Box-and-whiskers plots were used to describe sGP73 levels, and the Mann-Whitney $\mathrm{U}$ test was employed to compare the group differences in sGP73 levels. Receiver operating characteristic (ROC) curves were used to identify the optimal cutoff value of sGP73 for HCC diagnosis, and the areas under the curves (AUC) were compared 
Table I. Associations between sGP73 levels and clinicopathological characteristics of 462 patients with hepatocellular carcinoma.

\begin{tabular}{|c|c|c|c|c|}
\hline \multirow[b]{2}{*}{ Characteristics } & \multirow[b]{2}{*}{ Total, $\mathrm{n}$} & \multicolumn{2}{|c|}{ sGP73 level, n } & \multirow[b]{2}{*}{ P-value } \\
\hline & & Low & High & \\
\hline Number of patients & 462 & 228 & 234 & \\
\hline Age, years & & & & 0.483 \\
\hline$<50$ & 196 & 93 & 103 & \\
\hline$\geq 50$ & 266 & 135 & 131 & \\
\hline Sex & & & & 0.228 \\
\hline Male & 420 & 211 & 209 & \\
\hline Female & 42 & 17 & 25 & \\
\hline $\begin{array}{l}\text { ECOG performance } \\
\text { status }\end{array}$ & & & & $<0.001$ \\
\hline 0 & 119 & 82 & 37 & \\
\hline 1 & 284 & 132 & 152 & \\
\hline 2 & 47 & 12 & 35 & \\
\hline 3 & 8 & 2 & 6 & \\
\hline 4 & 4 & 0 & 4 & \\
\hline BCLC stage & & & & $<0.001$ \\
\hline 1 & 92 & 65 & 27 & \\
\hline 2 & 33 & 21 & 12 & \\
\hline 3 & 310 & 138 & 172 & \\
\hline 4 & 27 & 4 & 23 & \\
\hline Tumor number & & & & $<0.001$ \\
\hline 1 & 214 & 129 & 85 & \\
\hline 2 & 57 & 35 & 22 & \\
\hline$\geq 3$ & 191 & 64 & 127 & \\
\hline Tumor size, $\mathrm{cm}$ & & & & $<0.001$ \\
\hline$\leq 5$ & 188 & 115 & 73 & \\
\hline$>5$ & 274 & 113 & 161 & \\
\hline Satellite lesion & & & & $<0.001$ \\
\hline No & 257 & 156 & 101 & \\
\hline Yes & 205 & 72 & 133 & \\
\hline Vascular invasion & & & & $<0.001$ \\
\hline No & 203 & 125 & 78 & \\
\hline Yes & 259 & 103 & 156 & \\
\hline Tumor grade & & & & 0.311 \\
\hline 1 & 3 & 3 & 0 & \\
\hline 2 & 54 & 34 & 20 & \\
\hline 3 & 9 & 34 & 2 & \\
\hline Unknown & 396 & & & \\
\hline $\begin{array}{l}\text { Lymph node } \\
\text { metastasis }\end{array}$ & & & & 0.009 \\
\hline No & 307 & 165 & 142 & \\
\hline Yes & 155 & 63 & 92 & \\
\hline Distant metastasis & & & & $<0.001$ \\
\hline No & 394 & 212 & 182 & \\
\hline Yes & 68 & 16 & 52 & \\
\hline
\end{tabular}

Table I. Continued.

\begin{tabular}{|c|c|c|c|c|}
\hline \multirow[b]{2}{*}{ Characteristics } & \multirow[b]{2}{*}{ Total, $\mathrm{n}$} & \multicolumn{2}{|c|}{ sGP73 level, n } & \multirow[b]{2}{*}{ P-value } \\
\hline & & Low & High & \\
\hline Child-Pugh class & & & & $<0.001$ \\
\hline A & 339 & 197 & 142 & \\
\hline B & 100 & 29 & 71 & \\
\hline $\mathrm{C}$ & 23 & 2 & 21 & \\
\hline Etiology & & & & 0.200 \\
\hline $\mathrm{HBV}$ & 427 & 213 & 214 & \\
\hline $\mathrm{HCV}$ & 4 & 3 & 1 & \\
\hline $\mathrm{HBV}$ and $\mathrm{HCV}$ & 2 & 2 & 0 & \\
\hline Other & 29 & 11 & 18 & \\
\hline $\begin{array}{l}\text { Cirrhosis } \\
\text { background }\end{array}$ & & & & 0.99 \\
\hline No & 99 & 48 & 51 & \\
\hline Yes & 363 & 178 & 185 & \\
\hline AFP level, ng/ml & & & & $<0.001$ \\
\hline$<400$ & 262 & 149 & 113 & \\
\hline$\geq 400$ & 200 & 79 & 121 & \\
\hline Number of patients & 462 & 228 & 234 & \\
\hline
\end{tabular}

sGP73, serum Golgi protein 73; ECOG, Eastern Cooperative Oncology Group; BCLC, Barcelona-Clinic Liver Cancer; HBV, hepatitis B virus; $\mathrm{HCV}$, hepatitis B virus; $\mathrm{AFP}, \alpha$-fetoprotein. ${ }^{\mathrm{a}} \chi^{2}$ test.

using the $\mathrm{Z}$ test. The correlation between sGP73 and clinicopathological variables in patients with HCC was assessed by the $\chi^{2}$ test. The Kaplan-Meier method was employed to estimate the survival rate, and the log-rank test was used to assess the difference between curves. A Cox proportional hazards model was utilized to evaluate the prognostic value of multiple factors on survival rates. $\mathrm{P}<0.05$ was considered to indicate a statistically significant difference. Statistical analyses were performed using SPSS version 17.0 (SPSS, Inc., Chicago, IL, USA) and MedCalc version 9.0 (MedCalc, Mariakerke, Belgium).

\section{Results}

Comparison of sGP73 concentrations. The median concentrations of sGP73 in patients with HCC, liver cirrhosis and healthy controls were 18.7 (IQR, 5.7-45.6), 18.5 (IQR, 5.31-41.8), and 0 (IQR, 0-8.5) ng/ml, respectively. As shown in Fig. 1, the sGP73 levels in the HCC and cirrhosis patients were markedly higher than those in healthy controls $(\mathrm{P}<0.001)$. However, no significant difference was identified between the HCC and cirrhosis groups $(\mathrm{P}=0.632)$.

Sensitivity and specificity of $S$ GP73 and AFP for HCC diagnosis. ROC curve analysis was used to define optimal cutoff values as well as the sensitivity and specificity of sGP73 and AFP in differentiating HCC and cirrhosis. The AUC for sGP73 was 0.51 [95\% confidence interval (CI), 0.46-0.56], with a sensitivity of $27.79 \%$ and a specificity of $77.96 \%$ at the 


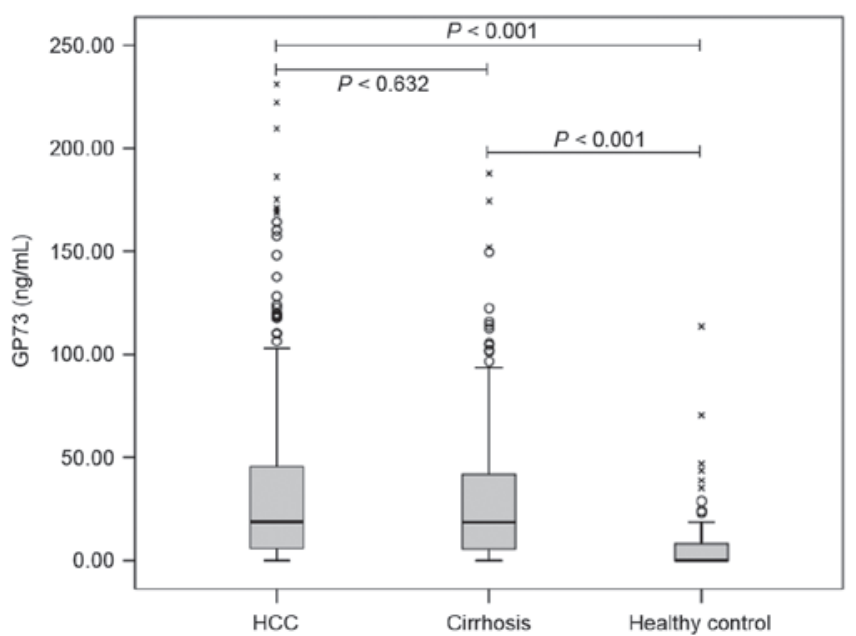

Figure 1. Serum GP73 levels in patients with hepatocellular carcinoma or liver cirrhosis, and healthy controls. Serum GP73 was detected by quantitative ELISA. Circular points indicate outliers and crosses indicate the extremes. GP73, Golgi protein 73.

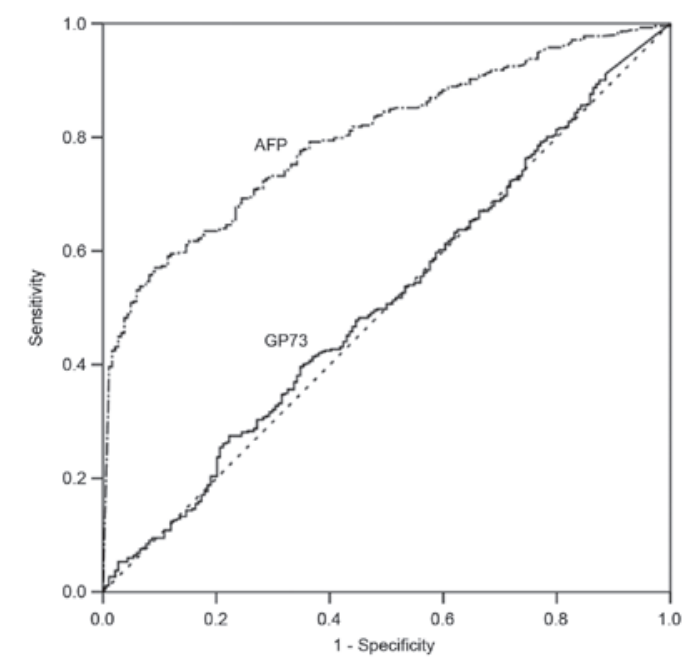

Figure 2. Receiver operating characteristic curves of serum GP73 and AFP for the diagnosis of hepatocellular carcinoma. GP73 had an inferior area under the curve compared with AFP (0.51 vs. 0.80; P<0.001). GP73, Golgi protein 73; AFP, $\alpha$-fetoprotein.

optimal cut-off point of $43.95 \mathrm{ng} / \mathrm{ml}$. The AUC for AFP was 0.80 (95\% CI, 0.77-0.83), with a sensitivity of $57.36 \%$ and a specificity of $90.96 \%$ at the optimal cutoff point of $93.27 \mathrm{ng} / \mathrm{ml}$ (Fig. 2). AFP had a significantly greater AUC than sGP73 for the diagnosis of $\mathrm{HCC}(\mathrm{P}<0.001)$.

Serum GP73 levels and HCC clinicopathological characteristics. To investigate the association between sGP73 levels and the clinicopathological characteristics of HCC, an optimal cutoff point of sGP73 for predicting OS was generated by ROC analysis. A sGP73 concentration $>18.74 \mathrm{ng} / \mathrm{ml}$ was defined as high-level and $\leq 18.74 \mathrm{ng} / \mathrm{ml}$ as low-level (AUC, 0.745; 95\% CI, 0.695-0.790). According to the cut-off point, high levels of sGP73 were observed in $50.6 \%$ (234/462) of patients with HCC.

As shown in Table I, high levels of sGP73 were significantly correlated with poor Eastern Cooperative Oncology

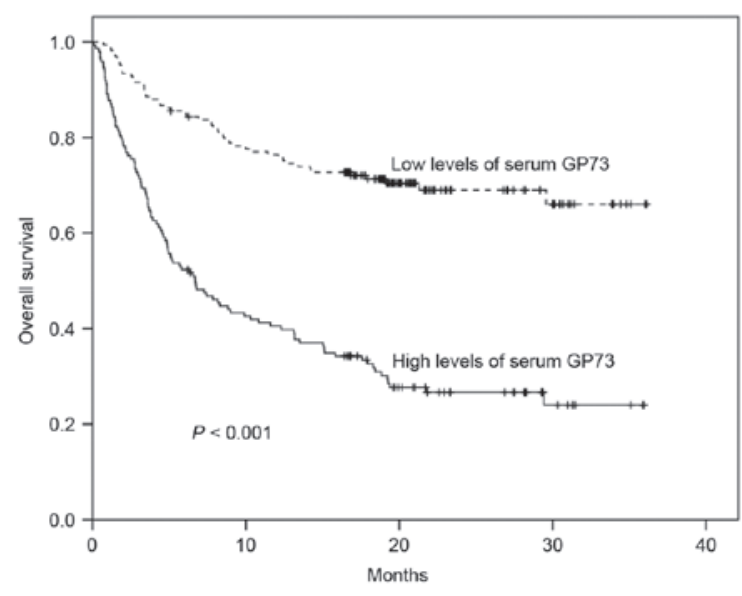

Figure 3. Kaplan-Meier survival curves for overall survival time according to serum GP73 levels in 313 patients with hepatocellular carcinoma. High serum GP73 levels were associated with decreased overall survival time compared with low levels of serum GP73. GP73, Golgi protein 73.

Group (ECOG) performance status $(\mathrm{P}<0.001)$, advanced Barcelona-Clinic Liver Cancer (BCLC) stage $(\mathrm{P}<0.001)$, multiple lesions $(\mathrm{P}<0.001)$, tumor size $>5 \mathrm{~cm}(\mathrm{P}<0.001)$, satellite lesions $(\mathrm{P}<0.001)$, vascular invasion $(\mathrm{P}<0.001)$, lymph node metastasis $(\mathrm{P}=0.009)$, distant metastasis $(\mathrm{P}<0.001)$, high Child-Pugh score $(\mathrm{P}<0.001)$, and AFP $\geq 400 \mathrm{ng} / \mathrm{ml}(\mathrm{P}<0.001)$. Other characteristics, including age, sex, etiology and liver cirrhosis background, were not significantly correlated with sGP73 level (all P>0.05).

Serum GP73 levels and HCC prognosis. Among the 462 HCC patients, complete follow-up information was available in 313 patients. The median follow-up time was 16.7 months (range, 0.07-36.13 months). During the follow-up, 157 patients $(50.2 \%)$ succumbed to tumor progression. Among the 128 early-stage HCC patients who received curative treatment, $48(37.5 \%)$ suffered tumor relapses, and $15(11.7 \%)$ ultimately succumbed to disease recurrence.

As shown in Fig. 3, high levels of sGP73 were associated with a significantly reduced 1-year OS rate compared with low levels of sGP73 (40.5 vs. $75.8 \%$; P<0.001). Poor OS was also associated with poor ECOG performance status $(\mathrm{P}<0.001)$, advanced BCLC stage $(\mathrm{P}<0.001)$, multiple lesions $(\mathrm{P}<0.001)$, tumor size $>5 \mathrm{~cm}(\mathrm{P}<0.001)$, satellite lesions $(\mathrm{P}<0.001)$, vascular invasion $(\mathrm{P}<0.001)$, lymph node metastasis $(\mathrm{P}<0.001)$, distant metastasis $(\mathrm{P}<0.001)$, high Child-Pugh score $(\mathrm{P}<0.001)$, and AFP $\geq 400 \mathrm{ng} / \mathrm{ml}(\mathrm{P}<0.001)$ (Table II). Importantly, Cox multivariate analysis confirmed that high levels of sGP73 constituted an independent poor prognostic factor for OS [hazard ratio (HR), 2.004; 95\% CI, 1.373-2.925; P<0.001; Table II]. In addition, ECOG performance status (HR, 1.421; 95\% CI, 1.070-1.886; $\mathrm{P}=0.015$ ), tumor size (HR, 2.400; 95\% CI, 1.309-4.400; $\mathrm{P}=0.005)$, vascular invasion (HR, 2.397; 95\% CI, 1.368-4.199; $\mathrm{P}=0.002$ ), and Child-Pugh class (HR, 1.920; 95\% CI, 1.426-2.587; $\mathrm{P}<0.001)$ were also negative independent predictors for OS. However, BCLC stage, tumor number, satellite lesions, lymph node metastasis, distant metastasis and AFP levels were not independent prognostic factors for OS (Table II). 


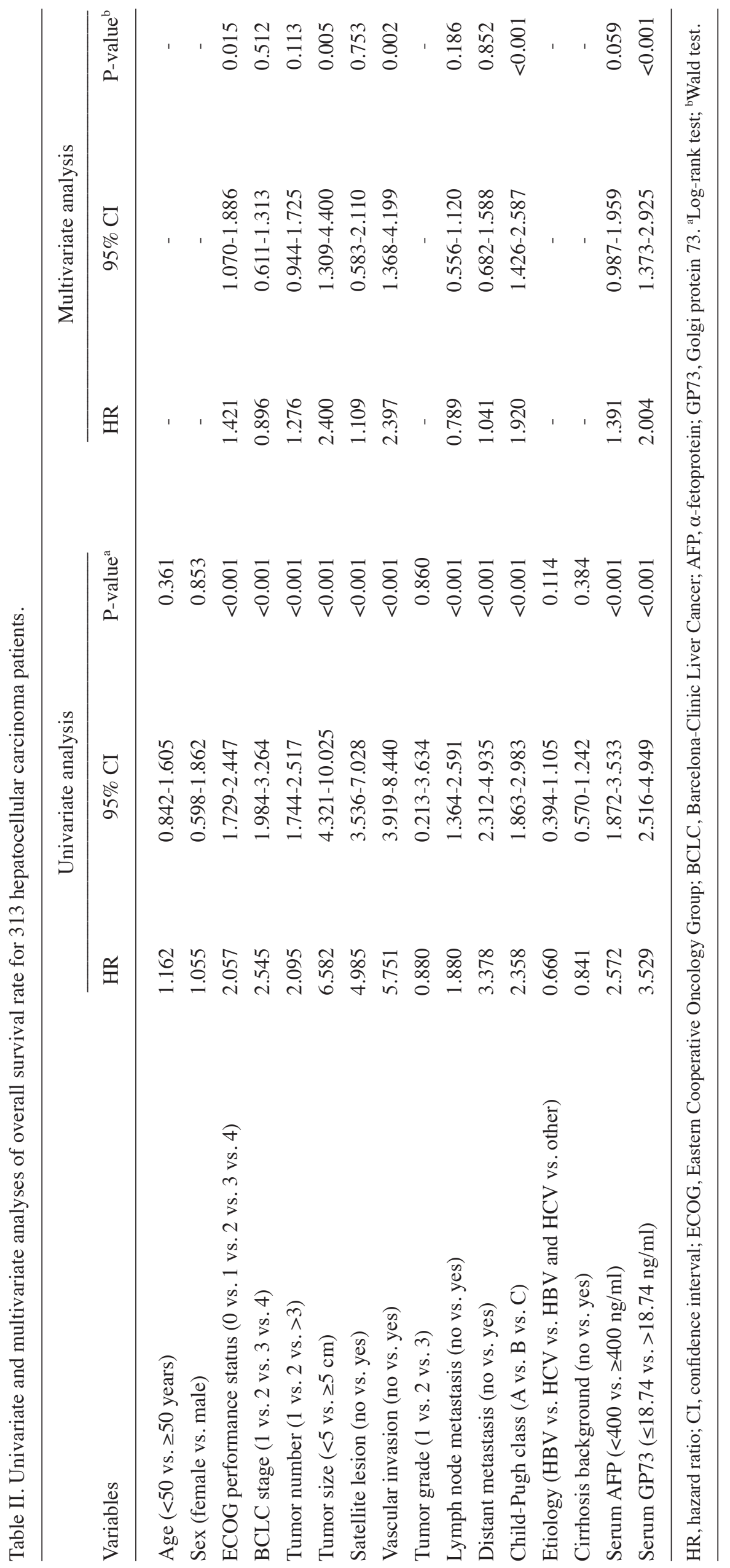




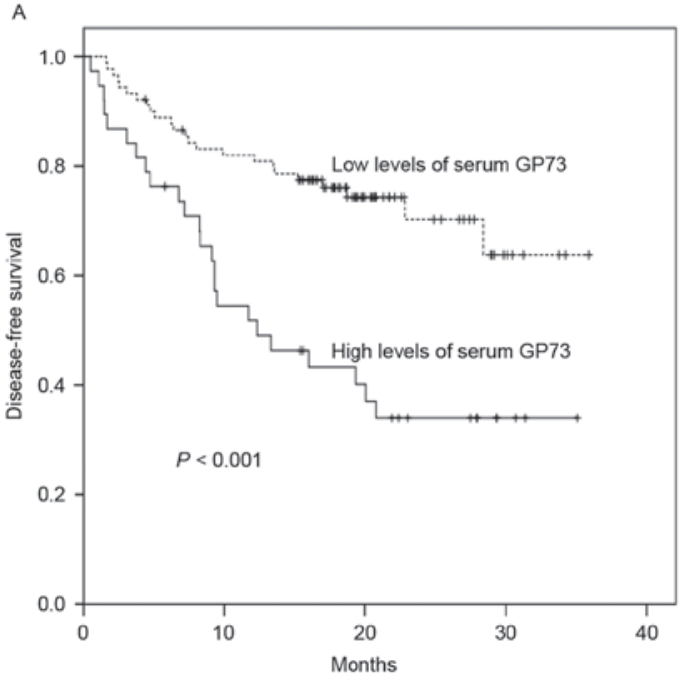

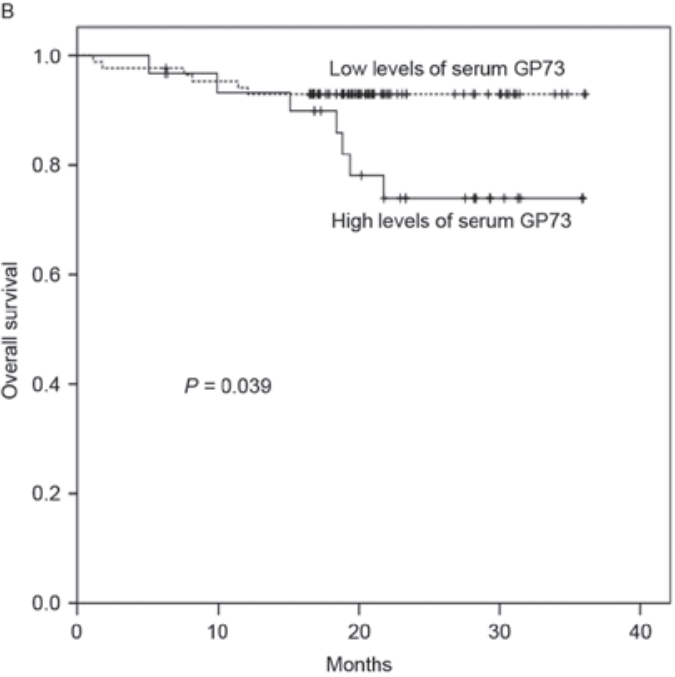

Figure 4. Kaplan-Meier survival curves for disease-free and overall survival times according to serum GP73 levels in 128 patients with hepatocellular carcinoma who underwent curative therapy. High levels of serum GP73 were associated with reduced (A) disease-free and (B) overall survival times. GP73, Golgi protein 73 .

To investigate the predictive value of sGP73 for HCC recurrence, the follow-up data of $128 \mathrm{HCC}$ patients who underwent curative treatment was subjected to survival analysis. As shown in Table III, in the univariate analysis, poor ECOG performance status $(\mathrm{P}=0.012)$, advanced BCLC stage $(\mathrm{P}=0.018)$, multiple lesions $(\mathrm{P}=0.041)$, tumor size $>5 \mathrm{~cm}$ $(\mathrm{P}=0.009)$, satellite lesions $(\mathrm{P}=0.002)$, vascular invasion $(\mathrm{P}=0.030)$, and sGP73 ( $\mathrm{P}<0.001$; Fig. 4A) were associated with poor DFS. However, only Child-Pugh class (HR, 3.747; 95\% CI, 1.305-10.765; P=0.014) and sGP73 (HR, 4.664; 95\% CI, 2.426-8.968; $\mathrm{P}<0.001)$ were negative independent prognostic biomarkers for DFS in the multivariate analysis (Table III). Similarly, for OS in this group, tumor size $(\mathrm{P}=0.019)$, satellite lesions $(\mathrm{P}=0.001)$, Child-Pugh class $(\mathrm{P}=0.044)$, and sGP73 $(\mathrm{P}=0.039$; Fig. 4B) were negative predictors in the univariate analysis, whereas satellite lesions (HR, 25.202; 95\% CI, 1.639-387.455; $\mathrm{P}=0.021$ ), Child-Pugh class (HR, 3.726; $95 \%$ CI, 1.059-13.113; P=0.041), and sGP73 (HR, 4.026; 95\% CI, 1.104-14.686; $\mathrm{P}=0.035$ ) were independent prognostic factors in the multivariate analysis (Table III).

\section{Discussion}

In the present study, it was found that SGP73 levels measured by ELISA were markedly higher in patients with HCC and liver cirrhosis than in healthy controls, whereas the difference in sGP73 levels between HCC and liver cirrhosis was not statistically significant. Both the sensitivity and specificity of sGP73 for the diagnosis of HCC were inferior to those of AFP. However, high sGP73 levels were significantly associated with aggressive clinicopathological features and decreased OS and DFS times in HCC. Multivariate analysis confirmed that sGP73 was an independent prognostic factor for HCC.

It is not entirely surprising that the diagnostic value of sGP73 was not detected in the present study. Upregulated hepatocyte expression of GP73 has previously been reported in the liver tissues not only of patients with HCC, but also of those with other liver disease, including liver cirrhosis (12), chronic hepatitis, hepatic atypical hyperplasia (15), liver cell adenoma, and focal nodular hyperplasia (23). High tissue levels of GP73 have also been observed in adenocarcinomas of the prostate, colon and breast, in renal cell cancer (26), and in bile duct carcinoma (23), suggesting that GP73 is not a HCC-specific biomarker. Iftikhar et al (13) observed that hepatocyte GP73 expression was markedly upregulated in patients with acute hepatitis, and that the upregulation was reversible upon the resolution of the acute disease. A similar magnitude of increased GP73 expression was observed in fully established cirrhosis. Consistently, a recent study demonstrated that the levels of sGP73 were low in chronic HBV carriers without liver injury, but they were significantly elevated in patients with prominent hepatic injury and fibrosis, and the sGP73 levels were positively correlated with the severity of liver injury, indicating that the affected hepatocytes released more GP73 into the blood when liver injury was triggered (27). Therefore, the increase of sGP73 is a common feature of liver injury, regardless of the causes.

Although the sGP73 levels in HCC were similar to those in liver cirrhosis in the present study, a number of studies reported higher sGP73 levels in HCC than in liver cirrhosis (19-21), or higher sGP73 levels in liver cirrhosis than in $\operatorname{HCC}(15,25)$. This discrepancy may be partly due to the heterogeneity of the disease stage of patients with $\mathrm{HCC}$ or liver cirrhosis among different studies. More advanced stages of HCC or liver cirrhosis reflect more injured hepatocytes, resulting in higher sGP73 levels in HCC or cirrhosis. Different GP73 antibodies used for the ELISA test may be another reason for this discrepancy. GP73 ELISA kits are commercially available; however, the different sensitivities and specificities of the kits provided by different manufacturers may lead to different results.

Since sGP73 was initially identified, studies have focused on its role in the early diagnosis of HCC $(11,18-21)$. Few studies have investigated the association of sGP73 with the clinicopathological characteristics and prognosis of HCC $(15,21)$. In the present study, it was identified that high levels of sGP73 were associated with aggressive tumor behavior and poor DFS and OS times, and that sGP73 was an independent negative prognostic factor 


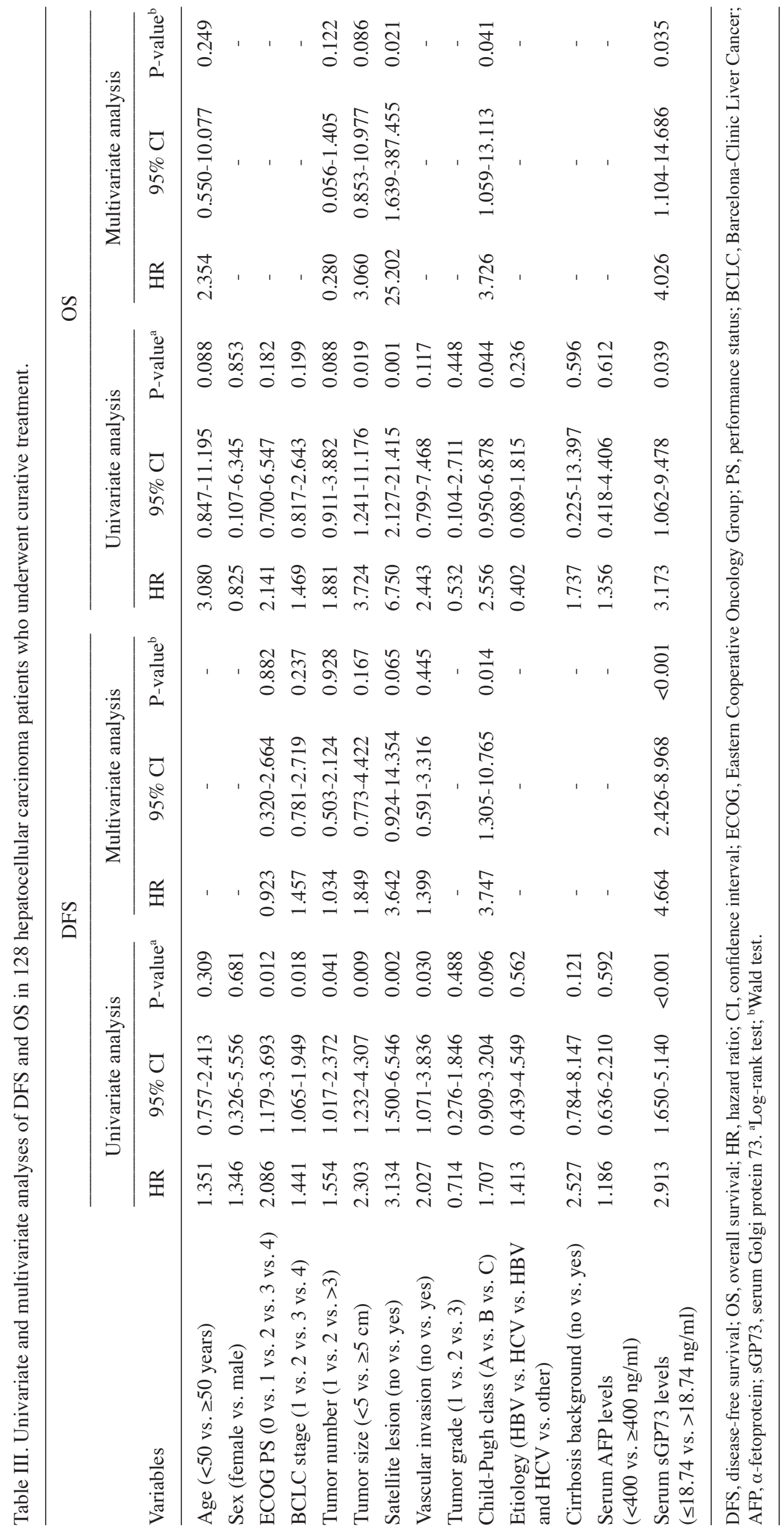


of HCC, which is consistent with the roles of overexpressed GP73 in HCC tissue (14). Significantly, with regard to predicting the risk of relapse and mortality, sGP73 had comparable HRs (4.664 and 4.026, respectively) with Child-Pugh class (3.747 and 3.726, respectively), and was superior to BCLC stage (Table III), suggesting that sGP73 has an important prognostic value in HCC. In HCC cell lines and xenograft tumors in mice, GP73 has been shown to promoted proliferation, migration, invasion and metastasis (28). However, the mechanism by which GP73 promotes tumor progression remains unclear. Recently, a study demonstrated that GP73 potentiated HCC cell invasion by upregulating matrix metalloproteinase-13 (29), supporting the role of GP73 in HCC as suggested by the present study.

In summary, the present study demonstrated that sGP73 was not a diagnostic marker but rather a prognostic factor for HCC. High levels of sGP73 were predictive of more aggressive features of HCC and poorer OS and DFS times; therefore, as a prognostic marker, sGP73 would be of great benefit for identifying the patients with a high risk of disease progression or recurrence, who may benefit from individualized therapy.

\section{Acknowledgements}

The present study was supported by the National Natural Science Foundation of China (grant no. 81372374 to Xiang-Yuan Wu), and the Science and Technology Foundation of Guangdong Province (grant no. 2011B031800014 to Min Dong).

\section{References}

1. Ferlay J, Shin HR, Bray F, Forman D, Mathers C and Parkin DM: Estimates of worldwide burden of cancer in 2008: GLOBOCAN 2008. Int J Cancer 127: 2893-2917, 2010.

2. Shariff MI, Cox IJ, Gomaa AI, Khan SA, Gedroyc W and Taylor-Robinson SD: Hepatocellular carcinoma: Current trends in worldwide epidemiology, risk factors, diagnosis and therapeutics. Expert Rev Gastroenterol Hepatol 3: 353-367, 2009.

3. Zeng H, Zheng R, Guo Y, Zhang S, Zou X, Wang N, Zhang L, Tang J, Chen J, Wei K, et al: Cancer survival in China, 2003-2005: A population-based study. Int J Cancer 136: 1921-1930, 2015.

4. Ercolani G, Grazi GL, Ravaioli M, Del Gaudio M, Gardini A, Cescon M, Varotti G, Cetta F and Cavallari A: Liver resection for hepatocellular carcinoma on cirrhosis: Univariate and multivariate analysis of risk factors for intrahepatic recurrence. Ann Surg 237: 536-543, 2003

5. Debruyne EN and Delanghe JR: Diagnosing and monitoring hepatocellular carcinoma with alpha-fetoprotein: New aspects and applications. Clin Chim Acta 395: 19-26, 2008.

6. Aoyagi Y, Suzuki Y, Isemura M, Nomoto M, Sekine C, Igarashi K and Ichida F: The fucosylation index of alpha-fetoprotein and its usefulness in the early diagnosis of hepatocellular carcinoma. Cancer 61: 769-774, 1988.

7. Nakagawa T, Seki T, Shiro T, Wakabayashi M, Imamura M, Itoh T, Tamai T, Nishimura A, Yamashiki N, Matsuzaki K, et al: Clinicopathologic significance of protein induced vitamin $\mathrm{K}$ absence or antagonist II and alpha-fetoprotein in hepatocellular carcinoma. Int J Oncol 14: 281-286, 1999.

8. Giardina MG, Matarazzo M, Varriale A, Morante R, Napoli A and Martino R: Serum alpha-L-fucosidase. A useful marker in the diagnosis of hepatocellular carcinoma. Cancer 70: 1044-1048, 1992.

9. Zhu ZW, Friess H, Wang L, Abou-Shady M, Zimmermann A, Lander AD, Korc M, Kleeff J and Büchler MW: Enhanced glypican-3 expression differentiates the majority of hepatocellular carcinomas from benign hepatic disorders. Gut 48: 558-564, 2001.

10. Giannelli G, Marinosci F, Sgarra C, Lupo L, Dentico P and Antonaci S: Clinical role of tissue and serum levels of SCCA antigen in hepatocellular carcinoma. Int J Cancer 116: 579-583, 2005 .
11. Mao Y, Yang H, Xu H, Lu X, Sang X, Du S, Zhao H, Chen W, $\mathrm{Xu}$ Y, Chi T, et al: Golgi protein 73 (GOLPH2) is a valuable serum marker for hepatocellular carcinoma. Gut 59: 1687-1693, 2010.

12. Kladney RD, Cui X, Bulla GA, Brunt EM and Fimmel CJ: Expression of GP73, a resident Golgi membrane protein, in viral and nonviral liver disease. Hepatology 35: 1431-1440, 2002

13. Iftikhar R, Kladney RD, Havlioglu N, Schmitt-Gräff A, Gusmirovic I, Solomon H, Luxon BA, Bacon BR and Fimmel CJ: Disease- and cell-specific expression of GP73 in human liver disease. Am J Gastroenterol 99: 1087-1095, 2004.

14. Bao YX, Cao Q, Yang Y, Mao R, Xiao L, Zhang H, Zhao HR and Wen $\mathrm{H}$ : Expression and prognostic significance of golgiglycoprotein 73 (GP73) with epithelial-mesenchymal transition (EMT) related molecules in hepatocellular carcinoma (HCC). Diagn Pathol 8: 197, 2013.

15. Shan SG, Gao YT, Xu YJ, Huang Y, Zhang Q, Zhai DK, Li JB, Wang FM, Jing X, Du Z and Wang YJ: Gradually increased Golgi protein 73 expression in the progression of benign liver diseases to precancerous lesions and hepatocellular carcinoma correlates with prognosis of patients. Hepatol Res 43: 1199-1210, 2013.

16. Bachert C, Fimmel C and Linstedt AD: Endosomal trafficking and proprotein convertase cleavage of cis Golgi protein GP73 produces marker for hepatocellular carcinoma. Traffic 8: 1415-1423, 2007

17. Block TM, Comunale MA, Lowman M, Steel LF, Romano PR, Fimmel C, Tennant BC, London WT, Evans AA, Blumberg BS, et al: Use of targeted glycoproteomics to identify serum glycoproteins that correlate with liver cancer in woodchucks and humans. Proc Natl Acad Sci USA 102: 779-784, 2005.

18. Marrero JA, Romano PR, Nikolaeva O, Steel L, Mehta A, Fimmel CJ, Comunale MA, D'Amelio A, Lok AS and Block TM: GP73, a resident Golgi glycoprotein, is a novel serum marker for hepatocellular carcinoma. J Hepatol 43: 1007-1012, 2005.

19. Shi Y, Chen J, Li L, Sun Z, Zen L, Xu S, Zhang Y and Zhang L: A study of diagnostic value of golgi protein GP73 and its genetic assay in primary hepatic carcinoma. Technol Cancer Res Treat 10: 287-294, 2011

20. Xu WJ, Guo BL, Han YG, Shi L and Ma WS: Diagnostic value of alpha-fetoprotein-L3 and Golgi protein 73 in hepatocellular carcinomas with low AFP levels. Tumour Biol 35: 12069-12074, 2014.

21. Hou SC, Xiao MB, Ni RZ, Ni WK, Jiang F, Li XY, Lu CH and Chen BY: Serum GP73 is complementary to AFP and GGT-II for the diagnosis of hepatocellular carcinoma. Oncol Lett 6: 1152-1158, 2013

22. Gu Y, Chen W, Zhao Y, Chen L and Peng T: Quantitative analysis of elevated serum Golgi protein-73 expression in patients with liver diseases. Ann Clin Biochem 46: 38-43, 2009.

23. Riener MO, Stenner F, Liewen H, Soll C, Breitenstein S, Pestalozzi BC, Samaras P, Probst-Hensch N, Hellerbrand C, Müllhaupt B, et al: Golgi phosphoprotein 2 (GOLPH2) expression in liver tumors and its value as a serum marker in hepatocellular carcinomas. Hepatology 49: 1602-1609, 2009.

24. Bröker ME, Ijzermans JN, Witjes CD, van Vuuren HJ and de Man RA: The predictive value of Golgi protein 73 in differentiating benign from malignant liver tumors. PLoS One 9: e100187, 2014

25. Tian L, Wang Y, Xu D, Gui J, Jia X, Tong H, Wen X, Dong Z and Tian Y: Serological AFP/Golgi protein 73 could be a new diagnostic parameter of hepatic diseases. Int J Cancer 129: 1923-1931, 2011.

26. Kristiansen G, Fritzsche FR, Wassermann K, Jäger C, Tölls A, Lein M, Stephan C, Jung K, Pilarsky C, Dietel M and Moch H: GOLPH2 protein expression as a novel tissue biomarker for prostate cancer: Implications for tissue-based diagnostics. $\mathrm{Br}$ J Cancer 99: 939-948, 2008.

27. Xu Z, Liu L, Pan X, Wei K, Wei M, Liu L, Yang H and Liu Q: Serum Golgi protein 73 (GP73) is a diagnostic and prognostic marker of chronic HBV liver disease. Medicine (Baltimore) 94: e659, 2015.

28. Chen X, Wang Y, Tao J, Shi Y, Gai X, Huang F, Ma Q, Zhou Z, Chen H, Zhang H, et al: mTORC1 Up-Regulates GP73 to promote proliferation and migration of hepatocellular carcinoma cells and growth of xenograft tumors in mice. Gastroenterology 149: 741-52.e14, 2015.

29. Jin D, Tao J, Li D, Wang Y, Li L, Hu Z, Zhou Z, Chang X, Qu $C$ and Zhang H: Golgi protein 73 activation of MMP-13 promotes hepatocellular carcinoma cell invasion. Oncotarget 6: 33523-33533, 2015. 